\title{
An Investigation into the Relation between the Technique of Movement and Overload in Step Aerobics
}

\author{
Alicja Rutkowska-Kucharska, ${ }^{1}$ Katarzyna Wysocka, ${ }^{2}$ Sławomir Winiarski, ${ }^{1}$ \\ Agnieszka Szpala, ${ }^{1}$ and Małgorzata Sobera ${ }^{2}$ \\ ${ }^{1}$ Department of Biomechanics, University School of Physical Education in Wroclaw, Wroclaw, Poland \\ ${ }^{2}$ Department of Athletics and Gymnastics, University School of Physical Education in Wroclaw, Wroclaw, Poland \\ Correspondence should be addressed to Alicja Rutkowska-Kucharska; alicja.rutkowska-kucharska@awf.wroc.pl
}

Received 3 November 2016; Revised 27 January 2017; Accepted 31 January 2017; Published 27 February 2017

Academic Editor: Andrea Marinozzi

Copyright (c) 2017 Alicja Rutkowska-Kucharska et al. This is an open access article distributed under the Creative Commons Attribution License, which permits unrestricted use, distribution, and reproduction in any medium, provided the original work is properly cited.

\begin{abstract}
The aim of this research was to determine the features of a step workout technique which may be related to motor system overloading in step aerobics. Subjects participating in the research were instructors $(n=15)$ and students $(n=15)$ without any prior experience in step aerobics. Kinematic and kinetic data was collected with the use of the BTS SMART system comprised of 6 calibrated video cameras and two Kistler force plates. The subjects' task was to perform basic steps. The following variables were analyzed: vertical, anteroposterior, and mediolateral ground reaction forces; foot flexion and abduction and adduction angles; knee joint flexion angle; and trunk flexion angle in the sagittal plane. The angle of a foot adduction recorded for the instructors was significantly smaller than that of the students. The knee joint angle while stepping up was significantly higher for the instructors compared to that for the students. Our research confirmed that foot dorsal flexion and adduction performed while stepping up increased load on the ankle joint. Both small and large angles of knee flexion while stepping up and down resulted in knee joint injuries. A small trunk flexion angle in the entire cycle of step workout shut down dorsal muscles, which stopped suppressing the load put on the spine.
\end{abstract}

\section{Introduction}

Step aerobics is one of the most popular forms of activity in fitness clubs. Many researchers emphasize its positive influence on circulation and the respiratory system [1], endurance, and muscle strength $[2,3]$. Specifically, step workout is considered to improve bone density which is so important in the prevention of osteoporosis $[4,5]$. However, if the volume and intensity of step exercise is too excessive, it may lead to motor system overloading which may manifest in pain and damage to the ankle and knee joints and spine [6-8]. It has been confirmed that injuries can be found in $77 \%$ of instructors, while $52.9 \%$ of which suffer from injuries of the lower extremities [9].

According to the research conducted by Machado and Abarantes [10], the number of steps performed during a workout ranges from 3500 to 6000 . Considering the fact that instructors often conduct up to 20 hours of step aerobics classes a week, it may be assumed that the main reason for motor system overloading is the large number of steps and movement sequences performed during a workout. Load put on the motor system in professional sports or during regular physical activity can be evaluated on the basis of vertical ground reaction forces (vGRF). Publications on motor system loads in step aerobics have addressed and analyzed vGRF while performing step-up exercises at different heights [11] and the suppressing properties of step $[12,13]$ when performing steps with high- and low-impact technique, as well as a different frequency of steps [14]. Machado and Abarantes [10] indicated that step workout dominated by high-impact steps generates a load of 1.50-1.54 body weight (BW), while that with low-impact steps generates a load of 1.12-1.25 BW. Bezner et al. [11] confirmed in their research that vGRF increased as step height increased (from 1.6 BW at low- 
bench step-up to $1.76 \mathrm{BW}$ at high-bench step-up), while Fujarczuk et al. [15] proved that the increase in step height significantly reduces the loading rate and the vGRF. There are two phases distinguished in a step workout which differ in the vGRF values. The vGRF values are lower when stepping up a bench in comparison to those obtained while stepping down $[7,14,16]$. The frequency of stepping up and down the bench is another factor having an influence on the load. Since step classes are performed with music, the intensity changes along with beat per minute (bpm). Changes in the tempo force participants to change the frequency of their steps which has an influence on the values of the vGRF $[6,14,17$, 18]. This factor also influences the increase of electrical activity in the muscles of the lower extremities [17]. A conclusion drawn from the presented research is that bpm determines values of the vGRF, varying from 1.12 to $1.54 \mathrm{BW}$ when stepping up the bench to $2.7 \mathrm{BW}$ when stepping down. The vGRF values obtained by the researchers mentioned above were similar to those observed during natural gait and stair climbing [19]. It can be assumed that values of the vGRF do not lead to injuries in people performing step workout. Another parameter describing loads on motor system is peak moments of force at joints in the lower extremities [20]. Hsu et al. [21] compared the maximum hip, knee, and ankle joint force between low- and high-impact aerobics and stair climbing. Their study showed that in high-impact step aerobics the joint forces were about 2-3 times higher than stair climbing, while Bezner et al. [11] searched for correlations between the height of the step and muscle torque. There were no statistically significant differences in moment of force at any of the ankle $(119.9-147 \mathrm{Nm})$, knee $(71.5-86.9 \mathrm{Nm})$, or hip (205.4$241.3 \mathrm{Nm}$ ) joints with respect to increases in height of the step and frequency of movement.

We believe that one of the reasons for injuries, similar to professional sports, may be step workout technique. Research on the loads of motor system in sport confirm that movement technique may decrease the risk of injuries [22]. If a movement is performed in a technically correct way, then the ground reaction forces are dumped in the following order: ankle, knee, and hip joints. The ability to dump ground reaction force depends on the muscle force and, more precisely, on the muscle torque acting on the joints. Although the issue of loads and their assessment in aerobics have been tackled since the '90s, it still seems to be absorbing to researchers. Especially now, new forms of physical fitness have been created (exercises performed on core board platforms, e.g.) whose form resembles that of step aerobics. The issue of injury, especially that of the ankle joint, became significant due to instability of the surface used to perform these exercises [23].

According to our knowledge, a relationship between motor system overloading in step aerobics and movement technique has yet to be examined. So far, only one study has tried to determine the relationship between the vGRF and foot position while stepping up a bench [6]. When the forefoot touched the step (finger technique), the researchers recorded 1.27-1.29 BW. A foot put flat on a step bench (flat technique) produced a load of 1.27-1.30 BW. When the heels were positioned on the bench, the first generated a load of
1.23-1.24 BW (heel technique). The authors did not observe any statistically significant differences between the techniques. Comprehensive description of step workout technique, similar to the description of extreme sport, is possible due to kinematic analysis. According to us, movement technique analysis based only on kinetic data is not sufficient since ground reaction forces are dumped in many joints of the motor system.

The aim of this research was to determine the features of step workout technique which may be related to motor system overloading in step aerobics. Knowing the step workout technique, we hypothesized that

(i) a risk of overloading of the ankle joint may result from the technique of setting the foot on a bench while stepping up,

(ii) knee joint injuries may result from the technique of stepping down with a small angle of knee joint flexion,

(iii) low back pain may be connected with the angle of a trunk flexion,

(iv) instructors, unlike the nonprofessionals, display a minimum risk of injury due to their movement technique.

\section{Materials and Methods}

The research was based on the analysis of students at the University School of Physical Education. The experiment was approved by the local ethics committee and conducted in accordance with the Declaration of Helsinki. Group one $(n=15)$ was comprised of the instructors (age 22.1 \pm 0.4 years, body mass $63.14 \pm 9.8 \mathrm{~kg}$, body height $168.9 \pm 4.0 \mathrm{~cm}$, BMI $22.19 \pm 3.6)$ who conducted step aerobics classes in fitness clubs. Group two $(n=15)$ was comprised of nonprofessionals students (age $23.0 \pm 0.9$ years old, body mass $63.1 \pm 9.13 \mathrm{~kg}$, body height $166.8 \pm 2.9 \mathrm{~cm}$, BMI $22.64 \pm 2.9$ ) who had not taken part in step aerobics classes before. The participants of the study were selected on the basis of their morphological body parameters, age, and lack of injuries of the motor system.

Before testing, the subjects participated in a standardized warm-up and stretching lasting 15 minutes. The motor task was to perform barefoot 12 basic steps at a tempo of 132 beats per minute (bpm).

Each motor task was repeated 3 times. The pulse of each subject was monitored in order to eliminate the effect of fatigue. If the heart rate did not obtain its resting values, the subject did not perform the next series of tasks. Further analysis was conducted on 339 basic steps selected from each group.

The basic step involved stepping with the right foot up the bench, bringing the left foot up, stepping down with the right foot, bringing the left foot back down, and then repeating the sequence [24]. No arm movements were added. When a tested subject performed the final step down, she was asked to remain there for the next 5 seconds so her body weight (BW) could be determined. 


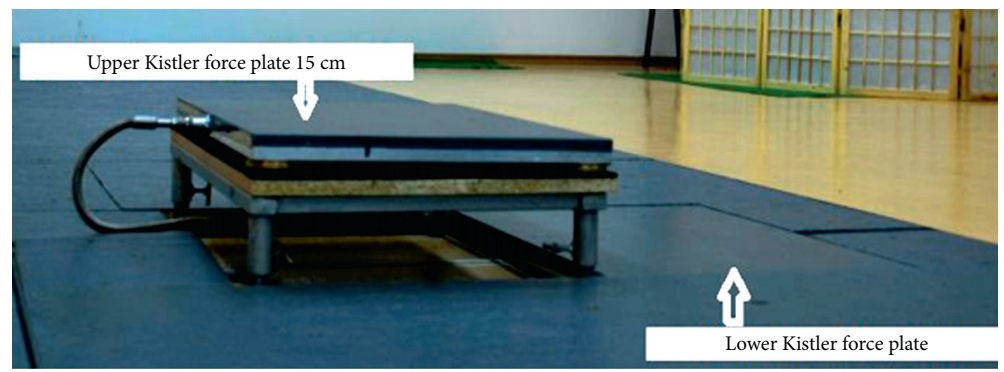

Figure 1: Experimental set-up. The first plate was embedded in the floor surface, and the second was fixed to a stable platform $15 \mathrm{~cm}$ above the first plate.

Kinematic and kinetic data was collected with the use of the BTS SMART system, which was comprised of 6 calibrated infrared video cameras at the frequency of $120 \mathrm{~Hz}$ and resolution of $640 \times 480 \mathrm{px}$. Additionally, the researchers used two Kistler 9286AA-A plates at the frequency of $1 \mathrm{kHz}$ and size of $600 \times 400 \mathrm{~mm}$, integrated with the gait analysis system. The first plate was embedded in the floor surface, while the second was fixed to a stable platform $15 \mathrm{~cm}$ above the first plate (Figure 1). The study was carried out at the Laboratory of Biomechanical Analysis, which is certified by the ISO Quality Certificate Number 1374-b/3/2009, PNEN ISO9001:2009.

The test subjects had a set of reflective markers attached to their skin at the following anatomical points: spinous processes of the seventh cervical vertebra, left and right acromion, anterior superior iliac spine (left and right), center of the sacrum at the height of the anterior superior iliac spine, greater trochanter of the femur (right and left), lateral epicondyle of the femur (right and left), head of the fibula (caput fibule, right and left), lateral ankle (lateral malleolus, right and left), head of the fifth external metatarsal bone (right and left), and tuber calcanei of the right and left limb and markers placed on rods: at the thigh coplanar with the lateral epicondyle of the femur and greater trochanter (right and left) and at the shin coplanar with head of the fibula and lateral malleolus (right and left).

The movement was divided into cycles. A cycle started when the foot was placed on the upper plate and ended when the same foot touched the bottom plate. Due to the course of the vertical ground reaction force component for the right foot, the entire task was divided into two cycles: stepping up and stepping down.

The following variables were analyzed: $F_{y}$-maximum for the vertical ground reaction force (vGRF), $F_{z}$-maximum for the anteroposterior ground reaction force (a-pGRF), and $F_{x}$-maximum for the mediolateral ground reaction force (m-lGRF), angle of ankle flexion in the sagittal plane, angle of foot abduction and adduction in the transverse plane, flexion and extension angle of the knee joint, and angle of trunk flexion in the sagittal plane. The analysis was conducted on the angle values attained during the movement phase when the vGRF reached its maximum value. When taking the measurements of foot flexion range and angle and the angle of abduction and adduction, the researchers determined the zero degree angle for a foot positioned flat on the ground.
Negative values described foot adduction. Straight knee joints and the trunk positioned at the vertical axis determined the zero angle and were applied as reference to measure the range of movement in the knee and hip joints.

Statistical analysis was conducted with the use of Statistica 12.5 software. Data normality was verified by the onesample Kolmogorov-Smirnov test and homogeneity of variances by Levene's test. Nonparametric measures were used (Mann-Whitney test and Spearman's rank correlation coefficient). Moreover, means and standard deviation were calculated for all the measured variables. The results were considered significantly different when the probability was less than or equal to 0.05 ( $p \leq 0.05)$.

\section{Results and Discussion}

Comparison of the GRF values attained for the three planes was conducted between the instructors and nonprofessionals. Statistically significant differences were only observed for the a-pGRF in the phase of stepping down from the upper plate and the m-lGRF in the phase of stepping up (Table 1).

Table 2 presents mean values of the kinematic parameters applied to compare the stepping up the bench technique of the instructors and nonprofessionals. Statistically significant differences were observed between the groups for the foot adduction angle. Values attained for both phases of movement showed that the nonprofessionals, in comparison to the instructors, adduced foot to a greater extent. Also, trunk flexion during stepping up and down was significantly higher in the nonprofessionals. However, the angle in the knee joint was significantly greater in the instructors' group.

In order to present the range of motion, as well as differences between the instructors and nonprofessionals, their values were normalized in the time of movement cycle (Figure 2). Foot dorsi-plantar flexion in the cycle was $24.5 \pm 2.2^{\circ}$ for the instructors and $22.1 \pm 1.7^{\circ}$ for the nonprofessionals. The angle of foot adduction-abduction was slightly smaller for the instructors $\left(11.6 \pm 3.5^{\circ}\right)$ than for the nonprofessionals $\left(11.9 \pm 3.1^{\circ}\right)$ throughout the entire movement cycle. The angle of knee joint flexion was significantly smaller for the instructors $\left(80.2 \pm 7.4^{\circ}\right)$ in comparison to the range of the nonprofessional group $\left(85.1 \pm 7.1^{\circ}\right)$. The range of trunk flexion was similar in both groups (instructors $8.7 \pm 1.3^{\circ}$ and nonprofessionals $8.7 \pm 1.4^{\circ}$ ). 
TABLE 1: Mean values and standard deviation (SD) of the GRF vector for the instructors and nonprofessionals recorded by the upper and lower Kistler force plate.

\begin{tabular}{lcccc}
\hline \multirow{2}{*}{ Variable } & \multicolumn{2}{c}{ Upper plate-stepping up } & \multicolumn{2}{c}{ Lower plate-stepping down } \\
& $\begin{array}{c}\text { Instructors } \\
\text { Mean } \pm \text { SD }\end{array}$ & $\begin{array}{c}\text { Nonprofessionals } \\
\text { Mean } \pm \text { SD }\end{array}$ & \multicolumn{2}{c}{$\begin{array}{c}\text { Instructors } \\
\text { Mean } \pm \text { SD }\end{array}$} \\
\hline$F_{y}[N]$ & $586.2 \pm 56.2$ & $637.3 \pm 83.0$ & $934.6 \pm 136.8$ & $1015.4 \pm 195.3$ \\
Relative $F_{y}(\mathrm{BW})$ & $1.0 \pm 0.1$ & $1.0 \pm 0.1$ & $1.6 \pm 0.2$ & $1.6 \pm 0.2$ \\
$F_{x}[N]$ & $-87.4 \pm 23.8$ & $-91.9 \pm-22.7$ & $118.3 \pm 49.1$ & $145.3 \pm 64.7$ \\
Relative $F_{x}(\mathrm{BW})$ & $-0.2 \pm-0.0$ & $-0.2 \pm-0.0$ & $0.21 \pm 0.09^{*}$ & $0.23 \pm 0.01^{*}$ \\
$F_{z}[N]$ & $-29.26 \pm-15.1$ & $-36.60 \pm-21.6$ & $26.89-25.3$ & $25.97-33.8$ \\
Relative $F_{z}(\mathrm{BW})$ & $-0.05 \pm-0.03^{*}$ & $-0.06 \pm-0.03^{*}$ & $0.05 \pm 0.04$ & $0.04 \pm 0.05$ \\
\hline
\end{tabular}

Note: ${ }^{*}$ statistically significant difference for $p \leq 0.05 ; F_{y}$-maximum for the vGRF component, $F_{x}-$ maximum for the a-pGRF component, $F_{z}-$ maximum for the $\mathrm{m}-\mathrm{lGRF}$ in the absolute $[N]$ and relative values (BW). The significance of differences was only tested for the relative values.

TABLE 2: Mean values and standard deviation (SD) angular variables obtained by the instructors and nonprofessionals when the vGRF is in its maximum value.

\begin{tabular}{|c|c|c|c|c|}
\hline \multirow{3}{*}{ Variable } & \multicolumn{2}{|c|}{ Upper plate-stepping up } & \multicolumn{2}{|c|}{ Lower plate-stepping down } \\
\hline & Instructors & Nonprofessionals & Instructors & Nonprofessionals \\
\hline & Mean \pm SD & Mean \pm SD & Mean \pm SD & Mean \pm SD \\
\hline Foot dorsal flexion $\left[{ }^{\circ}\right]$ & $16.9 \pm 3.8$ & $16.9 \pm 4.3$ & $19.7 \pm 5.0^{*}$ & $18.5 \pm 5.5^{*}$ \\
\hline Foot adduction $\left[{ }^{\circ}\right]$ & $-10.4 \pm-9.0^{*}$ & $-16.9 \pm-8.4^{*}$ & $-16.1 \pm-8.1^{*}$ & $-22.2 \pm-9.3^{*}$ \\
\hline Knee joint flexion $\left[{ }^{\circ}\right]$ & $47.5 \pm 7.0^{*}$ & $45.8 \pm 8.3^{*}$ & $17.5 \pm 8.0$ & $14.7 \pm 9.9$ \\
\hline Trunk flexion $\left[{ }^{\circ}\right]$ & $3.9 \pm 2.4^{*}$ & $5.8 \pm 2.6^{*}$ & $8.7 \pm 2.4^{*}$ & $9.5 \pm 1.9^{*}$ \\
\hline
\end{tabular}

*Statistically significant difference for $p \leq 0.05$.

Further analysis examined any correlation between the kinematic and kinetic parameters. Statistically significant correlations (level of significance $p \leq 0.05$ ) between the foot adduction and the a-pGRF while stepping up the bench were observed for both groups. Spearman's rank correlation coefficient for the nonprofessional group was 0.623 and for the instructors 0.538 . The angle of foot flexion of the instructors correlated (0.582) with the vGRF component.

Analysis of the vertical ground reaction force (vGRF) expressed in units of body weight (BW) while stepping up and down revealed similarities to values attained by other authors $[25,26]$. It should be emphasized that the examined subjects performed the barefoot movement on Kistler plates. Both the material of the bench and footwear suppress GRF which significantly decreases load on the motor system. Values of the vGRF presented in different publications as well as in ours were similar to those obtained during natural movement. Taking these two facts into consideration, it can be assumed the vGRF values cannot cause motor system overloads while performing step aerobics.

The anatomy of the ankle joint exposes it to injury during step workouts. The technique of placing the foot on the bench can be described by the anteroposterior (a-pGRF) and mediolateral (m-lGRF) ground reaction force, along with the range of movement in the sagittal and transverse planes. The analysis of the course of the a-pGRF confirmed that the technique of placing the foot on a bench was different to climbing upstairs. Walking upstairs begins with the forefoot striking the ground, followed by heel contact. In step aerobics, it is the heel which strikes the surface first, while the a-pGRF is twofold higher than climbing upstairs [27].
Moreover, the differences between these values are significantly different for the instructors and nonprofessionals. The a-pGRF values for the instructors were statistically significantly higher than that for the group of nonprofessionals. Relative values of the a-pGRF while stepping up the bench attained in our research were on average $0.20 \mathrm{BW}$. While Santos-Rocha et al. [14] recorded values of $0.30 \mathrm{BW}$. The difference observed between these findings may result from the fact that Santos-Rocha et al. [14] calculated the maximum value of the entire movement cycle (from stepping up to stepping down), whereas we have analyzed values of only the vertical component of the a-pGRF at the time of maximum vGRF.

Analysis of the mediolateral ground reaction force (mlGRF) showed that the subjects stepping up the bench put load on the midfoot and then transferred it toward the lateral part of the foot. Values of the m-lGRF ranged from 0.04 to $0.06 \mathrm{BW}$. Values of m-lGRF for climbing upstairs and walking on a flat surface do not usually exceed 0.045 BW [27]. Research in sport indicates that ankle joint muscles suppress maximal vGRF when it is the midfoot which makes contacts with the ground first $[28,29]$. This means that the lower the angle of plantar flexion in ground contact, the smaller the muscles' abilities to dump the GRF. This is vital in stepping down. While stepping down when the vGRF reached its maximum, both the instructors and nonprofessionals placed their foot in dorsiflexion, not in the plantar flexion, even though the ground contact with small plantar flexion. Moreover, in the instructors' group, there was a correlation between the angle of foot flexion and the vGRF values. These facts may also indicate a cause of ankle joint injuries. Analysis of the 


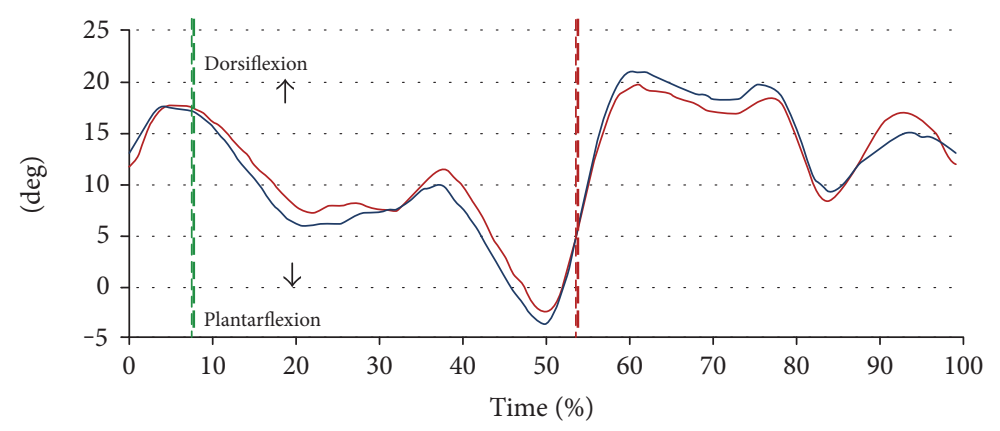

(a)

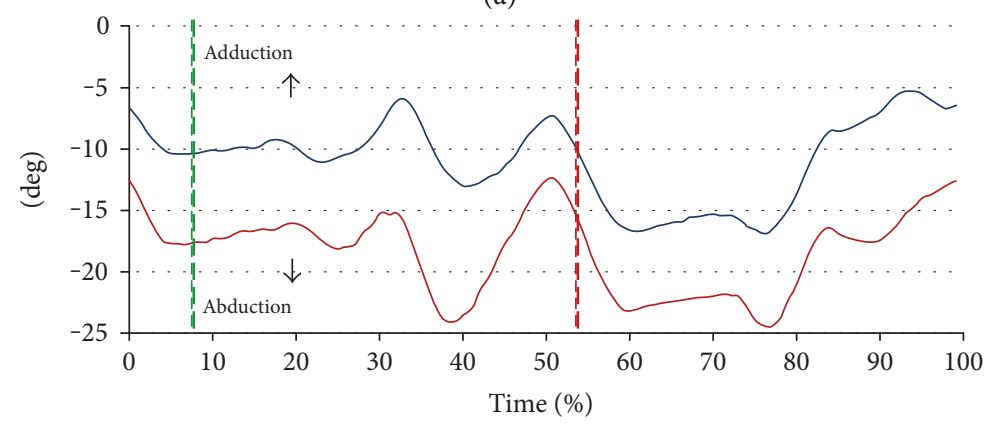

(b)

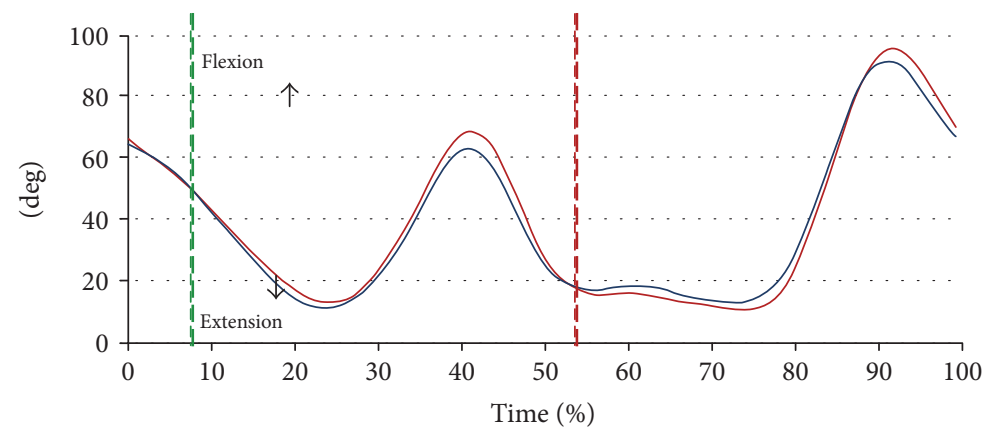

(c)

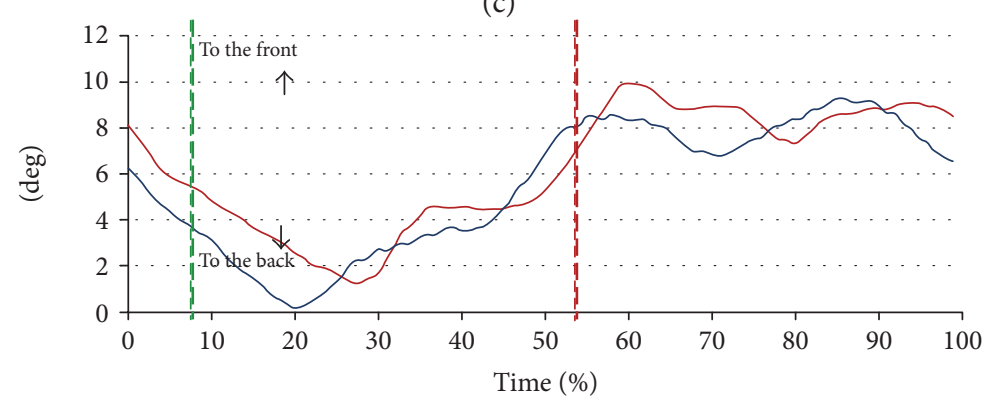

(d)

FIGURE 2: Mean parameters reflecting the technique of stepping up of the examined groups in a movement cycle where $y$-axis represents values of the angle $\left[^{\circ}\right]$. Blue-instructors, red-nonprofessionals. Vertical green line-vGRF during stepping up, red-during stepping down.

abduction and adduction angles while stepping up and down the bench may be another indicator of reasons for said injuries. The nonprofessionals placed their foot with greater adduction in comparison to the instructors. Such foot position may result in greater load on the midfoot joints and also medial head of the gastrocnemius muscle. The adduction angle observed in the nonprofessionals while stepping down exceeded the standard range of motion of $20^{\circ}$ [30]. Trew and Everett [31] reported that the majority of the population walk with positive foot angle (the foot is abducted in a straight line) of up to $30^{\circ}$ during normal gait. An asymmetric ankle joint load may lead to injuries in the nonprofessionals. The nonprofessionals place their foot on the bench differently than when walking upstairs. Gait analysis shows that when the foot first touches the ground, it is abducted by approximately 17 degrees and then returns to its neutral position corresponding to gait on a flat surface [32]. 
Values of the knee joint angle determined for stepping on the bench in the maximum vGRF were statistically significantly higher for the instructors $\left(47.5^{\circ}\right)$ than for the nonprofessionals $\left(45.8^{\circ}\right)$. Knee joint mechanics show that the joint angle of knee flexion and extension is related to the muscle moment arm of the knee joint flexors and extensors. This is why knee joint muscles performing a certain range of motion develop a higher or lower moment of muscle force. This means that when the angle of knee joint flexion is either large or small, the muscle moment arm is very short and the moment of force of knee flexors and extensors is also small. Stepping down with straight knees significantly decreases muscles' abilities to dump ground reaction forces and leads to an increased load on the articular surface. Small knee joint angles observed in our research while stepping down performed by both groups may be a reason for injuries. Our hypothesis is confirmed also by the study of van Husen et al. [33]. Their findings confirm that the load on the knee joint increases along with decreasing angle of knee joint flexion. Another reason for an injury may result from a significant angle of knee joint flexion when stepping up the bench (also short muscle moment arm) with the foot in adduction. Foot angle is mainly associated with the degree of rotation at the hip joint and the rotation between the tibia and femur [31]. Knee joint load in the external foot rotation leads to incorrect load distribution on the articular and menisci surfaces and injury in the medial collateral ligament. Such harmful load distribution on the knee joints repeated multiple times during step aerobics classes is probably a reason for pain and injuries of these joints. This is why parallel foot position in weight lifting is so important in order to avoid asymmetric load distribution on the knee joints. Research carried on by Valenzuela et al. [34] confirmed that external rotation of the foot during running reduces the load on the medial compartment of the knee.

The last body segment participating in suppressing vGRF is the spine. Research shows that the spine generates the highest moment of force when the angle of trunk flexion in the sagittal plane equals $60^{\circ}$ [35]. This conveys the idea that the lesser the angle of trunk flexion in sagittal plane, the greater the forces acting on the surface of the spinal joints and the greater the load on the paraspinal muscles. A small angle of trunk flexion for both stepping up and down (approx. $5^{\circ}$ when stepping up and $10^{\circ}$ when stepping down) point to a possible cause of low back pain in participants of step aerobics classes. The angle of trunk flexion was small for both the instructors and nonprofessionals which could have resulted from the specific type of the step workout. When conducting the class, instructors watch the participants in the mirror and maintain verbal and eye contact with them, the subjects follow their movements.

There is still a question which remains unanswered: how to decrease the risk of injury in step aerobics? Regarding the nonprofessionals, one should focus on positioning their feet while stepping up and down along the long body axis. This will decrease the risk of injury in the ankle and knee joints. Moreover, a greater angle of trunk flexion in the sagittal plane may diminish lower back pain. Overloading of the motor system in the instructors should be seen in the number of hours spent exercising. Perhaps, similar to professional athletes, the instructors should support the stabilizing function of muscles and the relatively weak collateral ligaments of the ankle joint by using prophylactic ankle supports.

\section{Conclusions}

Significant foot dorsiflexion observed when the heel strikes the ground while stepping up on the bench may be a cause of ankle joint overloading in instructors. In the case of nonprofessionals, the reason for such injuries may be ascribed to the angle of foot adduction and values of the vGRF components. Overloading of the knee joint observed in both groups resulted from the small angle of joint flexion in the external rotation of the shank in reference to femur. A small angle of trunk flexion while stepping up and down points to a possible cause of low back pain in participants of step aerobics classes.

\section{Competing Interests}

The authors declare that there is no conflict of interest regarding the publication of this paper.

\section{References}

[1] A. Kin-Isler, S. N. Kosar, and F. Korkusuz, "Effects on step aerobics on aerobic dancing on serum lipids and lipoproteins," Journal of Sports Medicine and Physical Fitness, vol. 41, no. 3, pp. 380-385, 2001

[2] J. M. Koenig, D. M. Jahn, T. E. Dohmeier, and J. W. Cleland, "The effect of bench step aerobics on muscular strength, power, and endurance," Journal of Strength and Conditioning Research, vol. 9, no. 1, pp. 43-46, 1995.

[3] W. J. Kraemer, M. Keuning, N. A. Ratamess et al., "Resistance training combined with bench-step aerobics enhances women's health profile," Medicine Science in Sports Exercise, vol. 33, no. 2, pp. 259-269, 2001.

[4] M. Lourdes Machado, H. Santos, F. Baptista, and A. Veloso, "Biomechanical effects of step exercise program on eldery women: support load effects analysis," 7th Annual Congress European College of Sport Science of Book of Abstract, 188 pages, Athens, July 24-28, 2002.

[5] C. H. Turner and A. G. Robling, "Designing exercise regiments to increase bone strength," Exercise and Sport Science Reviews, vol. 31, no. 1, pp. 45-50, 2003.

[6] A. Rutkowska-Kucharska and A. Szpala, "Relation between motor system loads and ground reaction force in step aerobic," Acta of Bioengineering and Biomechanics, vol. 6, supplement 1, pp. 293-297, 2004.

[7] M. C. Maybury and J. Waterfield, "An investigation into relation between step high and ground reaction forces step aerobics: a pilot study," British Journal of Sports Medicine, vol. 31, no. 2, pp. 109-113, 1997.

[8] H. N. Williford, L. A. Richards, M. Scharff-Olson, M. Brown, D. L. Blessing, and W. J. Duey, "Bench stepping and running in woman. Changes in fitness and injury status," Journal of 
Sports Medicine and Physical Fitness, vol. 38, no. 3, pp. 221226, 1998.

[9] V. du Toit and R. Smith, "Survey of aerobic dance injuries to the lower extremity in aerobics instructors," Journal of the American Podiatric Medical Association, vol. 91, no. 10, pp. 528-532, 2001.

[10] M. Lourdes Machado and J. Abarantes, "Basic step vs. power step: peak values of vertical GRF analysis," Proceedings of the XVI International Symposium on Biomechanics in Sports of Book of Abstract, pp. 514-517, Konstanz, July 21-25, 1998.

[11] S. A. Bezner, S. A. Chinworth, D. M. Drewlinger et al., "Step aerobics: a kinematic and kinetic analysis," Proceedings of the 11 International Symposium on Biomechanics in Sport, pp. 252-254, Amherst, Massachusetts, USA, 1993.

[12] G. J. Salem, S. R. Ward, and T. Q. Lee, "Regulation of plantar-foot kinetics during exercise on step benches with markedly different structural properties," Journal of Strength and Conditioning Research, vol. 14, no. 1, pp. 26-31, 2000.

[13] W. A. Skelly, L. A. Darby, and K. Phillips, "Physiological and biomechanical responses to three different landing surfaces during step aerobics," Journal of Exercise Physiology, vol. 6, no. 2, pp. 70-79, 2003.

[14] R. Santos-Rocha, A. Veloso, M. Lourdes Machado, M. João Valamatos, and C. Ferreira, "Peak ground and joint forces in step-exercise depending on step-pattern and stepping-rate," The Open Sports Sciences Journal, vol. 2, pp. 10-21, 2009.

[15] K. Fujarczuk, S. Winiarski, and A. Rutkowska-Kucharska, "Ground reaction forces in step aerobics," Acta of Bioengineering and Biomechanics, vol. 8, no. 2, pp. 111-118, 2006.

[16] A. Tsiokanos and E. Kellis, "Vertical ground reaction forces in step aerobic dance," 6th Annual Congress European College of Sports Science of Book of Abstract, 1101 pages, CologneGermany, July 24-28, 2001.

[17] R. Santos-Rocha, S. Franco, P. Correia, and A. Veloso, "Influence of music tempo on muscle participation level in step exercise," 5th Annual Congress European College of Sport Science of Book of Abstract, 644 pages, Jyvaskyla, Finland, July 19-23, 2000.

[18] R. Santos-Rocha, A. Veloso, H. Santos, S. Franco, and P. Pezarat-Correia, "Ground reaction forces of step exercise depending on step frequency and bench height," Scientific Proceedings of the XXIth International Symposium Biomechanics in Sports, pp. 156-158, Caceres, Spain, 2002.

[19] A. Stacoff, I. A. Kramers-de Quervain, G. Luder, R. List, and E. Stussi, "Ground reaction forces on stairs. Part II: knee implant patients versus normal," Gait and Posture, vol. 26, no. 1, pp. 48-58, 2007.

[20] D. Iwańska and C. Urbanik, "The sense of position and movement in the knee joint during voluntary movements," Acta of Bioengineering and Biomechanics, vol. 15, no. 3, pp. 11-17, 2013.

[21] H. C. Hsu, H. W. Wu, and Y. W. Chang, "Kinetic analysis of lower limbs in step aerobic dance," Gait and Posture, vol. 26, supplement 1, pp. S83-S84, 2007.

[22] A. Rutkowska-Kucharska, T. Bober, and R. Serafin, "Loads acting on the locomotor system in sports," Biocybernetics and Biomedical Engineering. Part III Biomechanics of Sports. Biomechanical Analysis of Loads in Sports, M. Nałęcz, Ed., EXIT, Warsaw, 2004.

[23] M. Karczewska, A. Madej, A. Sadowska, A. Mastalerz, and C. Urbanik, "Comparison of the ground reaction forces during the basic step on the core board platform at various levels of the stability," Acta of Bioengineering and Biomechanics, vol. 18, no. 4, pp. 63-70, 2016.

[24] J. Milewska, S. Jaroszczuk, and A. Rutkowska-Kucharska, "Step aerobics and rhythm of movement of the upper and lower limbs," Human Movement, vol. 14, no. 1, pp. 70-75, 2013.

[25] J. Lythe, J. Hunter, and P. Pfitzinger, Vertical Ground Reaction Force during Bench Step Aerobics. Comparison of Step Frequency and Step Style, UniSports Centre for Sport Performance, 2000.

[26] R. Santos-Rocha, R. Oliveira, and A. Veloso, "Osteogenic index of step-exercise depending on choreographic movements, session duration and stepping-rate," British Journal of Sports Medicine, vol. 40, no. 10, pp. 860-866, 2006.

[27] R. Riener, M. Rabuffetti, and C. Frigo, "Stair ascent and descent at different inclinations," Gait and Posture, vol. 15, no. 1, pp. 32-44, 2002.

[28] L. B. Dworak, J. Mączyński, and T. Wojtkowiak, "Dependency of dynamic overloads on the type of surface, on the basis of the acrobatic element standing backward somersault," Physical Education and Sport, vol. 3, pp. 33-40, 1996.

[29] J. Gorwa, L. B. Dworak, R. Michnik, and J. Jurkojc, "Kinematic analysis of modern dance movement "stag jump" within the context of impact loads, injury to the locomotor system and its prevention," Medical Science Monitor, vol. 20, pp. 1082-1089, 2014.

[30] J. E. Kotwick, "Biomechanics of the foot and ankle," Clinics in Sports Medicine, vol. 1, no. 1, pp. 19-34, 1982.

[31] M. Trew and T. Everett, Human Movement. An Introductory Text, Elsevier Churchill Livingstone, Edinburgh, 2005.

[32] S. Nadeau, B. McFadyen, and F. Malouin, "Frontal and sagittal plane analyses of the stair climbing task in healthy adults aged over 40 years: what are the challenges compared to level walking?” Clinical biomechanics, vol. 18, no. 10, pp. 950-959, 2003.

[33] M. van Husen, K. Peikenkamp, and K. Nicol, "Comparison of landings in handball and volleyball," 6th Annual Congress European College of Sports Science of Book of Abstract, 1225 pages, Cologne-Germany, July 24-28, 2001.

[34] K. A. Valenzuela, S. K. Lynn, G. J. Noffal, and L. E. Brown, "Acute effects of foot rotation in healthy adults during running on knee moments and lateral-medial shear force," Journal of Sport Science and Medicine, vol. 15, no. 1, pp. 50-56, 2016.

[35] V. M. Zatsiorsky, Kinetics of Human Motion, Human Kinetics, Leeds, United Kingdom, 2002. 


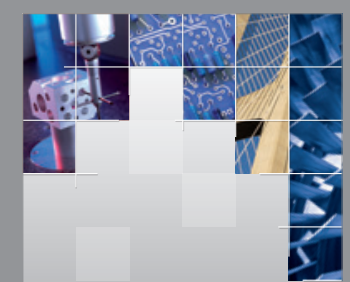

\section{Enfincering}
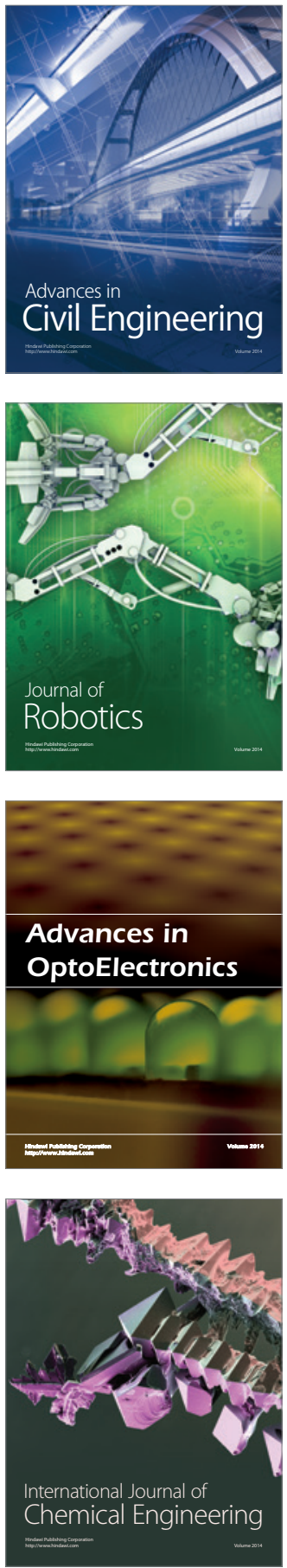

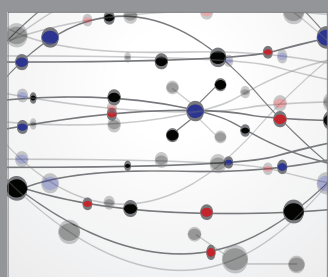

The Scientific World Journal

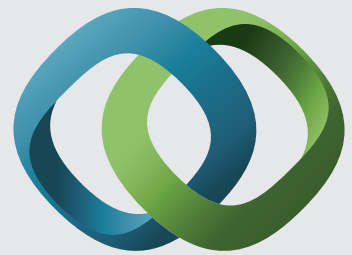

\section{Hindawi}

Submit your manuscripts at

https://www.hindawi.com
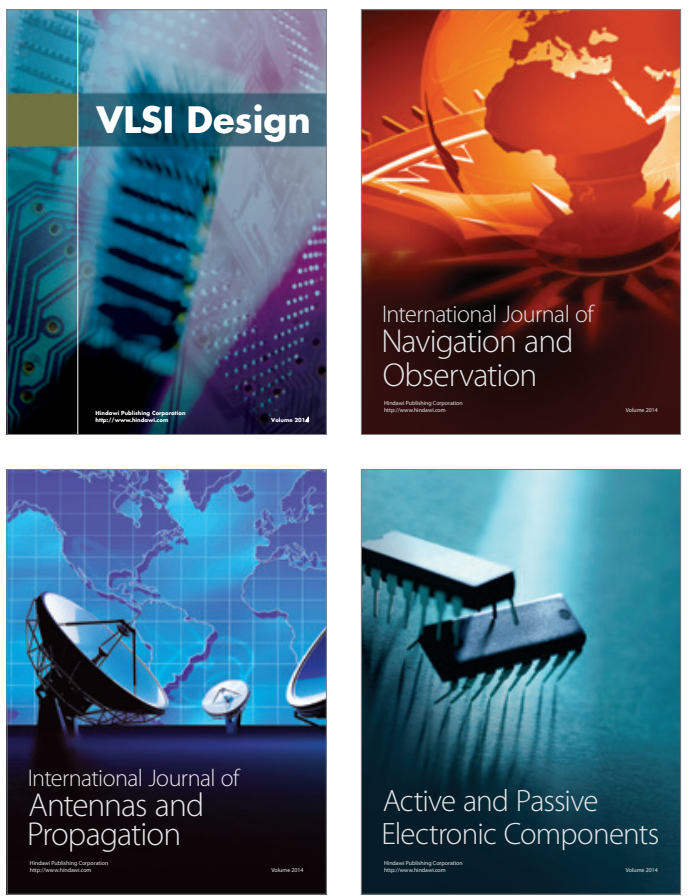
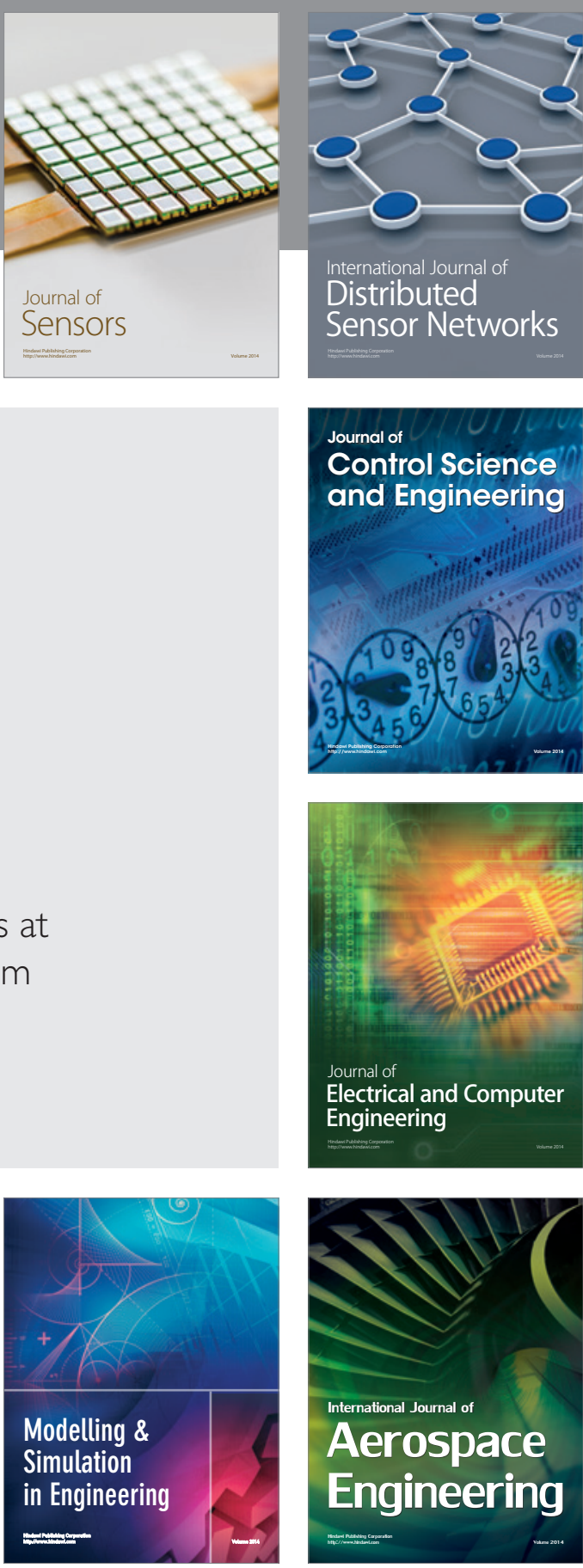

International Journal of

Distributed

Sensor Networks

$-$

Joumal of

Control Science

and Engineering
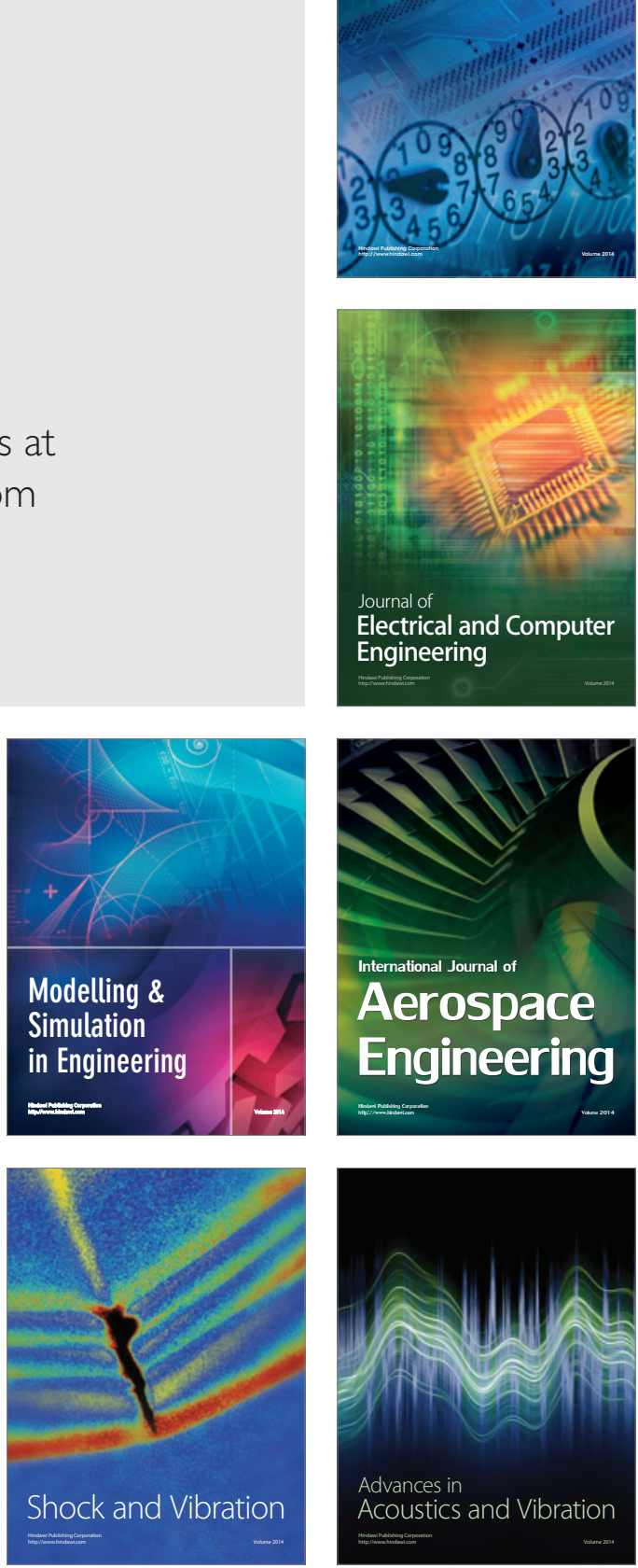